\title{
Detection and discrimination of subjective contours defined by offset gratings
}

\author{
RICK GURNSEY, MARTA IORDANOVA, and DAISY GRINBERG \\ Concordia University, Montréal, Québec, Canada
}

\begin{abstract}
Three experiments were conducted to refine our understanding of the mechanisms that encode subjective contours. In Experiment 1, discrimination thresholds (stimulus onset asynchronies [SOAs] yielding $81 \%$ correct) were measured in a backward masking paradigm for subjective contours defined by offset gratings. For large apertures, thresholds increased as carrier frequency increased. For the smallest aperture, thresholds were a U-shaped function of carrier frequency. Experiment 2 showed that these threshold results were generally consistent with the rated strength of the subjective contours. Experiment 3 showed that detection thresholds (SOAs yielding $81 \%$ correct) again increased with carrier spatial frequency, increased for obliquely oriented carriers, and, for a particular frequency and orientation of the carrier, were lower when the subjective contour was orthogonal to the carrier. All of these results are well explained by a two-stage process in which a second-layer filter integrates the responses of endstopped mechanisms to the terminators defining the subjective contour. In the model, the end-stopped mechanisms have low-pass sensitivity to carrier spatial frequency, and the sizes of the second-layer filters are proportional to the scale of the end-stopped mechanisms from which they draw their input.
\end{abstract}

Vision involves the recovery of properties of the physical world from their projections in the retinal images. Image contours are critical to this process, because they can arise from many important physical events (Marr, 1982). For example, image contours may be projections of lighting discontinuities (e.g., shadows or illuminant irregularities), material discontinuities (e.g., color or texture), disparities, and relative motions. An important class of discontinuity arises from abrupt changes in depthfor example, when one surface occludes another or two surfaces meet to form an edge. Although such discontinuities are often associated with luminance change, this is not always the case. There are conditions in which changes in local image geometry are the only clues to discontinuities in depth.

Subjective contours and texture boundaries are examples of image discontinuities that are not conveyed by luminance discontinuities. Subjective contours, in particular, have long been studied, precisely because the percept does not arise from a luminance discontinuity. Contour extraction is thought to be low level, in that it precedes identification of the scene events to which the contours correspond. Low-level constructions are generally thought to arise early in the visual system in a reflexive way that

This research was supported by NSERC and FCAR research grants to R.G. We thank Michael von Grünau and Frédéric Poirier for helpful discussions. We also acknowledge the very helpful comments of two anonymous reviewers. Correspondence concerning this article should be addressed to R. Gurnsey, Department of Psychology, Concordia University, 7141 Sherbrooke Street West, Montréal, PQ, H4B 1R6 Canada (e-mail: gurnsey@vax2.concordia.ca).

-Accepted by previous editor, Myron L. Braunstein is independent of an observer's current plans, beliefs, goals, expectations, or attentional state. Recent physiological evidence is consistent with the idea that subjective contours of the sort shown in Figure 1 are represented at a very low level in the geniculostriate stream. ${ }^{1}$ Thus, inasmuch as subjective contours are exemplars of occlusion contours, it may be argued that occlusion is a property that is extracted very early in the processing stream.

\section{MODELS OF SUBJECTIVE CONTOUR EXTRACTION}

It is widely believed that the perception of subjective contours is mediated by multiple layers of spatial filtering (e.g., Grossberg \& Mingolla, 1985; Gurnsey, Humphrey, \& Kapitan, 1992; Gurnsey \& von Grünau, 1997; von der Heydt \& Peterhans, 1989; Wilson \& Mast, 1993; Wilson \& Richards, 1992). The orientations of static subjective contours cannot be encoded explicitly in the outputs of linear spatial filters (Gurnsey \& von Grünau, 1997). The usual view is that, for subjective contours to be encoded, the input image is transformed in some way before the application of orientation-selective filters. Gurnsey and von Grünau suggested that the process described in Figure 1 (panels B and C) provides an adequate account of the extraction of subjective contours. (Gurnsey \& von Grünau also showed that such a mechanism could be elaborated so as to provide an explanation of the percepts arising from translating subjective contours.) Conceptually, there are two stages in the model. The first stage responds to line endings and has the kind of sensitivity found in single end-stopped cells in area V1 (Kato, Bishop, \& Orban, 1978). (In Figure 1, end-stopping is accomplished by taking the difference 
A

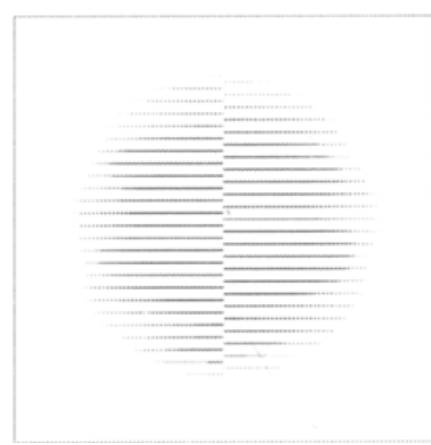

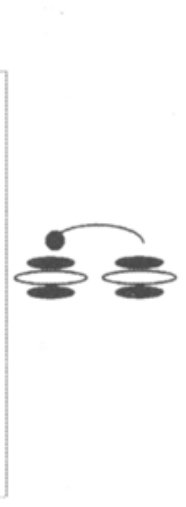

B

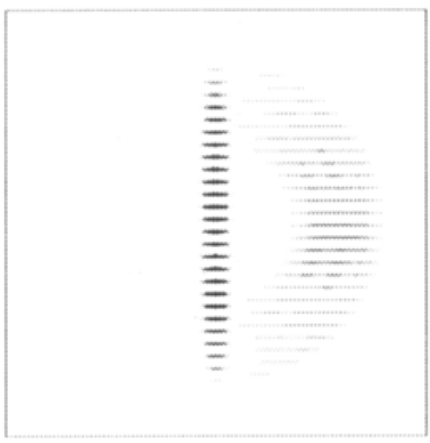

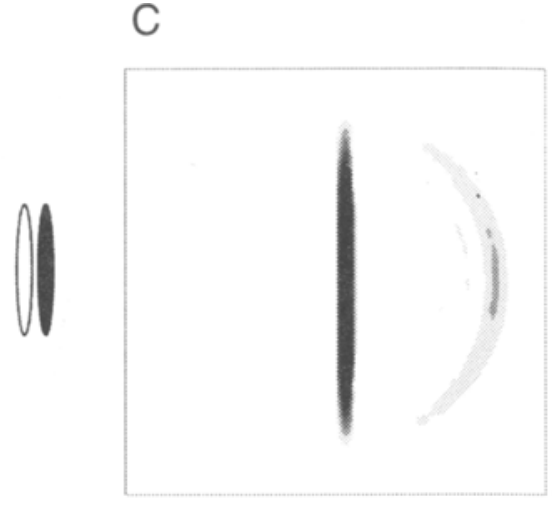

Figure 1. The two-stage model of subjective contour encoding. Panel A shows a subjective contour within a circular aperture. The process of extracting line endings is accomplished by taking the difference between the rectified responses of two filters that are displaced by a small amount along their long axes (panel B). (The convolution outputs in panels B-C have been half-wave rectified. White indicates zero response, and darker grays indicate stronger responses.) Panel $C$ shows the result of passing a large, vertically selective filter over the end-stopped representation. There is a very clear ridge of responses at the locus of the subjective contour.

between the half-rectified responses of two filters having the same orientation preference, offset in a direction parallel to their long axes. This is quite similar to the approach taken by Heitger, Rosenthaler, von der Heydt, Peterhans, \& Kubler, 1992, who differentiated the responses of orientation-selective filters in a direction parallel to the filter's preferred orientation.) A second-stage, orientation-selective filter is applied to the end-stopped representation to reveal locations of aligned end-stopped responses. The mechanism shows a particular sensitivity to subjective contours defined by offset gratings.

The general character of the model described in Figure 1 is widely accepted as a plausible hypothesis about how subjective contours are encoded. Its plausibility inheres partly in the fact that related models can explain texture segmentation (Bergen \& Landy, 1991; Gurnsey, Pearson, \& Day, 1996), the perception of non-Fourier motions (Wilson \& Mast, 1993), and the detection of symmetry (Gurnsey, Herbert, \& Kenemy, 1998). In the present paper, we address two questions. The first concerns the size of the second-layer mechanism and its relationship to the spatial frequency selectivity of the end-stopped mechanism from which it draws its input. The second question concerns the relationship between the orientation selectivity of the end-stopped mechanism and the second-layer, orientation-selective filter.

\section{GENERAL METHOD}

Experiments I and 3, described below, employ the same basic methodology. Stimulus presentation was followed by a mask at a variable stimulus onset asynchrony (SOA). Experiment 1 employed a two-alternative forced choice procedure in which the subjects had to determine whether the subjective contour presented on a given trial was left or right oblique. Experiment 3 employed a yes/no detection task in which subjective contours were either present or absent. The method of constant stimuli was employed for both tasks. For each stimulus condition, a Weibull function was fit to the data, using a maximum likelihood procedure. The SOA that yielded $81 \%$ correct was taken as the stimulus threshold.

\section{The Displays}

The stimuli were drawn in circular apertures (see Figure 2). Onepixel-wide lines $\left(\approx 0.037^{\circ}\right.$ of visual angle) of the carrier grating had a fixed contrast within a central region of the aperture. Outside this central region, the contrast dropped to zero over a range of 30 pixels $\left(\approx 1.1^{\circ}\right)$. The drop was a linear function of the distance from the diameter of the central region. The background intensity was approximately $25 \mathrm{~cd} / \mathrm{m}^{2}$, and the darkest lines had an intensity of $15 \mathrm{~cd} / \mathrm{m}^{2}$, yielding a Michelson contrast of about $25 \%$.

Each display was followed by a high contrast $(>90 \%)$ masking stimulus composed of $2 \times 2$ pixel $\left(0.074^{\circ} \times 0.074^{\circ}\right)$ black dots on a white background. The black dots were randomly positioned, and each pixel in the mask had a $5 \%$ chance of being black. The diameter of the mask was always 260 pixels $\left(9.62^{\circ}\right)$.

\section{EXPERIMENT 1 The Effects of Carrier Spatial Frequency and Contour Length}

The purpose of Experiment 1 was to determine the sensitivity of the visual system to subjective contours of different lengths, defined by offsets in gratings of different frequencies.

\section{Method}

Subjects. There were 6 subjects in the experiment. All were undergraduates in the Department of Psychology at Concordia University. None had any prior experience in psychophysical experiments. All the subjects reported normal or corrected-to-normal vision.

Apparatus and Materials. The stimuli were presented on an lkagami gray-scale monitor under the control of an Apple Quadra 950 computer. The look-up table (mapping digital-analog converter [DAC] values to gun intensity) was calibrated to be linear. The screen was viewed from a distance of $57 \mathrm{~cm}$, and a chinrest was used to stabilize head position.

The subjects were asked to discriminate between left- and rightoblique $\left( \pm 45^{\circ}\right)$ subjective contours defined by displacements of the carrier grating. There were five carrier spatial frequencies and three 
A

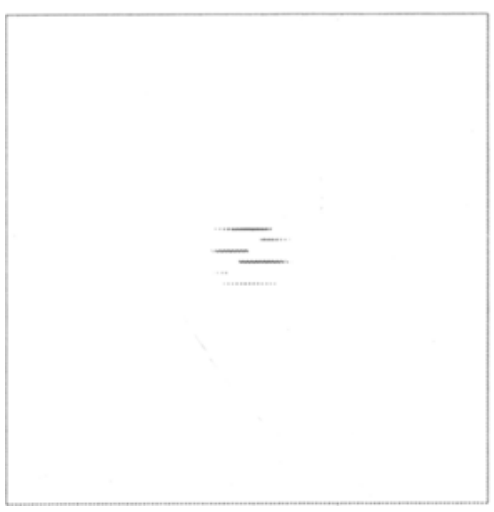

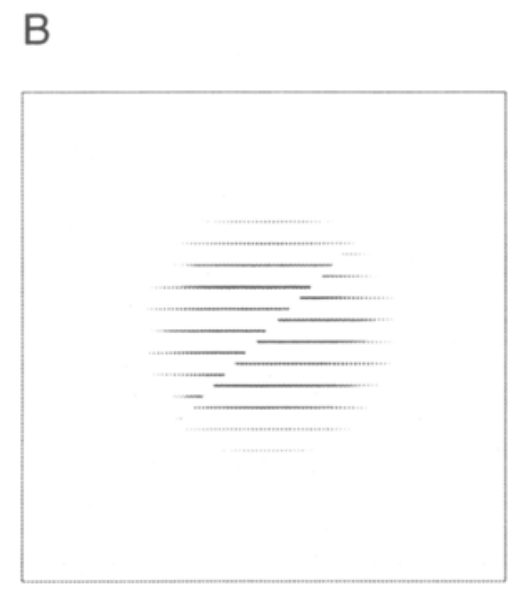

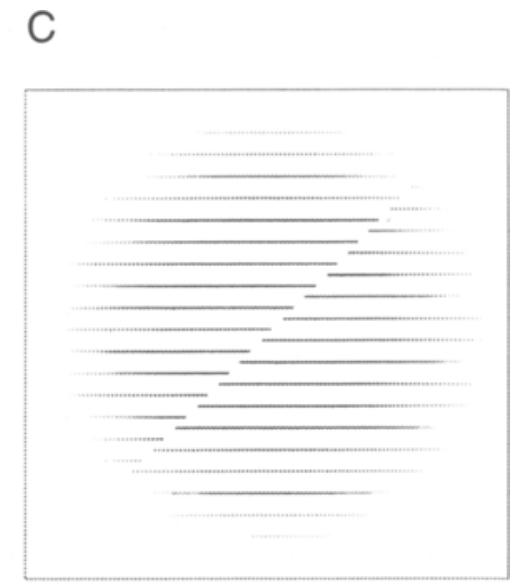

Figure 2. Examples of the stimuli used in Experiment 1. Panels $A-C$ show subjective contours embedded in apertures of $1.5^{\circ}, 4.0^{\circ}$, and $6.67^{\circ}$, respectively. In these examples, all the carriers have the same spatial frequency.

aperture sizes. The carrier spatial frequencies were $7.5,3.75,2.5$, 1.875 , and $1.5 \mathrm{cpd}$, corresponding to interline spacings of $4,8,12$, 16 , or 20 pixels, respectively. The three aperture sizes were, nominally, 40,120 , and 200 pixels, which corresponded to the central regions of the apertures having a constant contrast of $25 \%$. When viewed from $57 \mathrm{~cm}$, these sizes corresponded to $1.5^{\circ}, 4.0^{\circ}$, and $6.67^{\circ}$ of visual angle, respectively. Examples of the three aperture sizes for a fixed spatial frequency are given in Figure 2.

Procedure. There were 15 combinations of carrier spatial frequency (5) and aperture size (3). Each trial began with the presentation of a small, low-contrast fixation dot that remained on until a button on the computer keyboard was pressed to initiate the stimulus presentation. Following the buttonpress, 1 of the 15 stimulus displays was presented and remained on the screen for $30,45,60,75$, 90,105 , or $150 \mathrm{msec}$. Immediately following the offset of the stimulus, the mask appeared for $300 \mathrm{msec}$. Following each display, the participants indicated (via a buttonpress) whether they had perceived a left- or a right-oblique grating, and responses were scored as correct or incorrect. A block of trials consisted of 210 presentations ( 5 carrier spatial frequencies, 3 aperture sizes, 7 SOAs, and 2 contour orientations). Each subject performed 10 blocks of trials, for a total of 2,100 trials. For each combination of carrier spatial frequency and aperture size, a Weibull function was fit to the SOA data, using a maximum likelihood procedure. The threshold SOA $(\approx 81 \%$ correct) was taken as the dependent measure.

\section{Results and Discussion}

A two-factor, within-subjects analysis of variance (ANOVA) was performed on the data. The ANOVA revealed a significant main effect of aperture size $[F(2,10)=$ $15.12, p<.001]$, a significant main effect of carrier spatial frequency $[F(4,20)=10.06, p<.001]$, and a significant interaction of carrier spatial frequency and aperture size $[F(8,40)=9.09, p<.0001]$. All the effects remained significant after Geisser-Greenhouse correction for violations of the homogeneity of variances and covariances assumptions of the ANOVA (Keppel, 1991). Figure 3 summarizes the carrier spatial frequency by aperture size interaction.

At the highest carrier frequencies ( 7.5 and $3.75 \mathrm{cpd}$ ), performance was clearly independent of aperture size; performance was, therefore, limited only by the carrier spatial frequencies. For carrier frequencies of $2.5 \mathrm{cpd}$ and lower, performance depended on the size of the aperture. For the two largest apertures $\left(4.0^{\circ}\right.$ and $\left.6.67^{\circ}\right)$, thresholds increased steadily as a function of carrier frequency. For the smallest aperture $\left(1.5^{\circ}\right)$, thresholds showed a quadratic dependence on carrier spatial frequency; thresholds were high for low-frequency carriers, decreased as carrier frequency increased over the range of $1.5-3.75 \mathrm{cpd}$, and then increased from 3.75 to $7.5 \mathrm{cpd}$. This result suggests that a critical number of line endings is required to engage effectively the mechanism responsible for encoding the subjective contour. Once that critical number has been obtained, performance is only limited by the spatial frequency of the carrier grating. A model of this process is developed below.

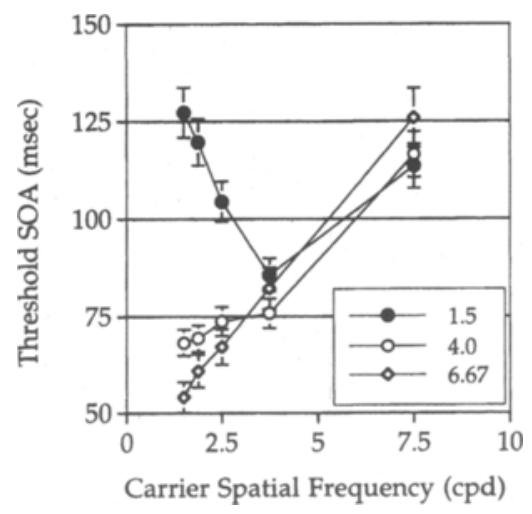

Figure 3. The results of Experiment 1. Threshold stimulus onset asynchrony (SOA) is plotted as a function of carrier spatial frequency for each of the three aperture sizes. For the two largest apertures $\left(4.0^{\circ}\right.$ and $\left.6.67^{\circ}\right)$, thresholds increased linearly with carrier frequency. For the smallest aperture $\left(1.5^{\circ}\right)$, thresholds first decreased with carrier frequency and then increased again as carrier frequency increased further. (Error bars indicate $\pm 1 S E M$.) 


\section{EXPERIMENT 2 Rated Strength of Subjective Contours}

Experiment 1 was intended to tap the properties of a subjective contour-detecting mechanism, such as the one shown in Figure 1. However, one may ask whether there is any evidence that the way in which aperture size and carrier frequency affect discrimination (in a masking experiment) would similarly affect the perception of subjective contours. This question is pertinent, because one could argue that the detection of terminators and of their positions in the aperture is sufficient to explain the results of Experiment 1 -that is, the results may not reflect the mediation of a mechanism such as the one shown in Figure 1. There are a number of difficulties with this approach to explaining the data, and we will deal with these in the General Discussion section. At this point, however, we address directly the effects of carrier frequency and aperture size on the perception of subjective contours.

\section{Method}

Subjects. There were 6 subjects in the experiment. All were undergraduate or graduate students in the Department of Psychology at Concordia University. All had prior experience in psychophysical experiments, but none had previously participated in experiments relating to subjective contours. None was aware of the results of Experiment I. All the subjects reported normal or corrected-tonormal vision.

Apparatus and Materials. The stimuli were presented on a 17in. Apple color monitor under the control of an Apple Power Macintosh 7100/80 computer. The look-up table (mapping DAC values to gun intensity) was calibrated to be linear. The screen was viewed from a distance of $57 \mathrm{~cm}$.

Procedure. The subjective contour stimuli in this experiment were exactly the same as those used in Experiment 1 . The subject's task was to rate the salience of each of the subjective contours that were presented to them. When the subjects were initially brought into the laboratory, they were shown a random subset of the 15 stimuli hav- ing different carrier frequencies and aperture sizes. Their attention was drawn to the fact that subjective contours were formed by the aligned terminators and that the strength or clarity of the subjective contours may vary as a function of carrier frequency and aperture size. At no time did the experimenter suggest how the strength or clarity of the subjective contours might vary. The subjects agreed that the different stimuli induced differing strengths of subjective contours.

Following this brief introduction, the subjects were told that they would be asked to rate the strength of a number of stimuli by assigning a number between 1 and 7 to each, where 7 indicated a very strong subjective contour and 1 indicated a weak or poor subjective contour. The subjects were then shown the entire set of 15 subjective contour stimuli in a random order and were asked to think about how they would rate each stimulus on the 7-point scale. The stimuli were presented for $1 \mathrm{sec}$ each, and the experimenter controlled the rate of presentation.

In the next phase, the subjects performed a practice run of the experiment, in which they were again presented with each of the 15 stimuli in a random order. Trials were initiated by the subject by pressing any key on the computer keyboard. They entered numbers between 1 and 7 (also on the computer keyboard) to indicate the strength of the subjective contour. The data from the practice run were not retained.

The experiment itself was exactly like the practice trials, except that there were four blocks of trials. Within a block, each of the stimuli was presented once, and the order of presentation was random.

\section{Results and Discussion}

For each subject, the four ratings for each display were averaged. The main objective was to relate the rating data to the exposure duration thresholds obtained in Experiment 1 . Two very different patterns of responses emerged. Four of the 6 subjects showed average responses (Figure 4) that corresponded remarkably well with the threshold results of Experiment 1 (cf. Figure 3; the results in Figure 3 and Figure 4A are inverses of one another, because Figure 3 represents thresholds and Figure 4 represents rated strength). For the two largest
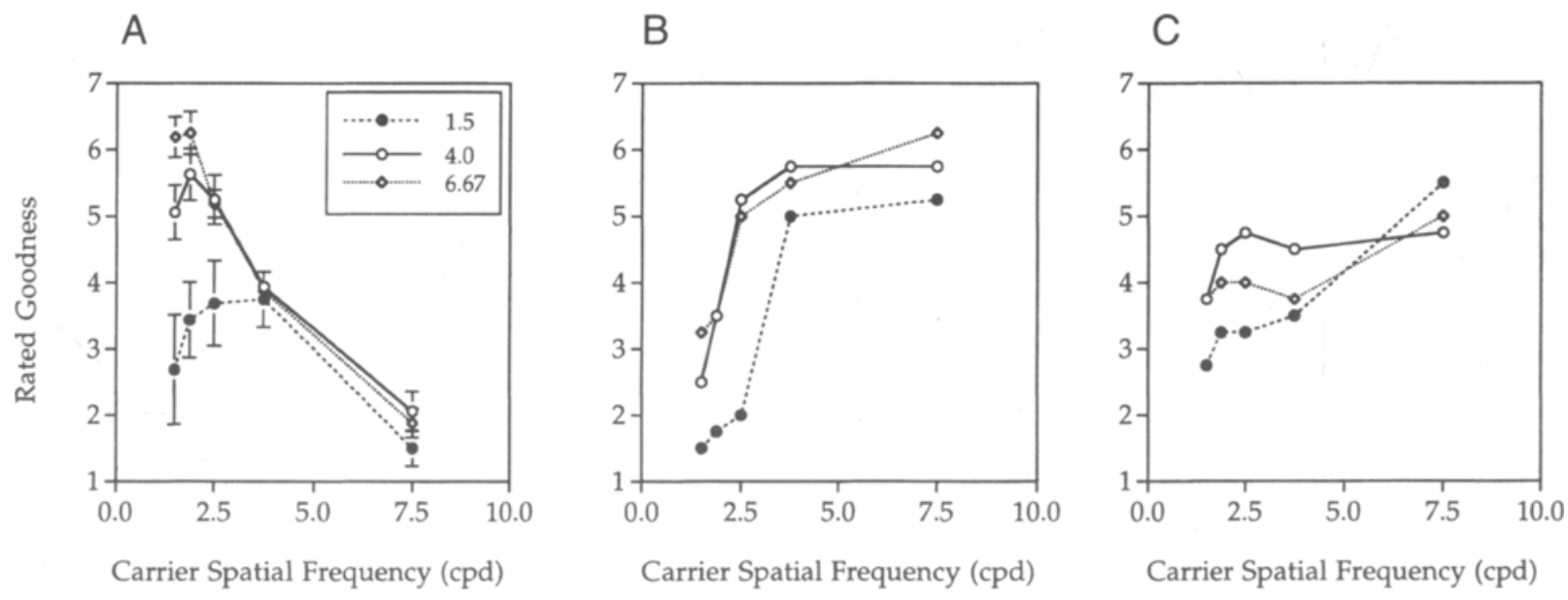

Figure 4. Results of Experiment 2. Panel A shows the averaged responses of 4 subjects as a function of carrier frequency and aperture size $\left(1.5^{\circ}, 4.0^{\circ}, 6.67^{\circ}\right)$. (Error bars indicate $\pm 1 S E M$.) These results are in excellent agreement with the threshold data shown in Figure 3. Panels $B$ and $C$ show the results for 2 subjects that diverged from the results shown in panel $A$. For these 2 subjects, the rated strength of the subjective contours increased with the carrier spatial frequency. 
aperture sizes, rated subjective contour strength showed an inverse relationship to carrier spatial frequency, independent of aperture size. For the smallest aperture size, ratings followed an inverted- $U$ function of carrier frequency. For these 4 subjects, it seems that ratings and thresholds can be explained by the responses of the same mechanisms. In other words, the two experimental procedures cross-validate each other.

Two of the 6 subjects responded very differently (Figures $4 \mathrm{~B}$ and $4 \mathrm{C}$ ). These subjects rated subjective contours defined in high-frequency carriers to be stronger than those in low-frequency carriers.

The results of Experiment 2 are mixed. Four of the 6 subjects gave rating responses that corresponded almost exactly in form with the threshold data obtained from the subjects in Experiment 1. This encourages the view that responses were made in terms of the same internal representations in the two experiments. On the other hand, the divergence in rating responses between our two subgroups of subjects is perplexing. If it is the case that, for the subjects shown in panels $B$ and $C$ of Figure 4, the internal representation of subjective contours is inverted, relative to the remaining subjects, we must conclude that there is a general lack of consistency in the way in which subjects perceive subjective contours.

It is possible, however, that the data in Figures $4 \mathrm{~B}$ and $4 \mathrm{C}$ reflect response strategies. Given a complex stimulus, a subject may have many representations available on which to base his or her response. In a rating experiment, the subject must decide how to relate these internal representations to rating responses. The two subgroups of subjects may have selected different internal representations on which to base their ratings. We would argue that 4 of the subjects in Experiment 2 based their rating responses on the same representation that governed thresholds in Experiment 1. The remaining subjects appear to have chosen a different strategy. However, it is unclear just what representation was involved in this latter choice. It should be pointed out that the SOA threshold methodology was used in Experiment 1 precisely because of concerns about the possible contaminating effects of strategic factors in rating data.

\section{EXPERIMENT 3 Effects of Carrier/Contour Orthogonality}

In Experiments 1 and 2, subjective contours were oriented $45^{\circ}$ to their carriers. There are several lines of evidence suggesting that this relationship may be suboptimal. Von der Heydt and Peterhans (1989) reported that contour cells in V2 responded best when the subjective contours were orthogonal to their carrier grating. Soriano, Spillman, and Bach (1996, Figure 8) created vertical subjective contours from the aligned terminations of gratings having different orientations. One grating was horizontal, and the other was of variable orientation. They found that when the orientation difference between the two gratings exceeded about $20^{\circ}$, the rated strength of the subjective contour diminished. This suggests that carrier orthogonality is an important determinant of subjective contour salience.

Experiment 3 addressed the question of whether subjective contours orthogonal to their carrier gratings are more easily detected than subjective contours that are oriented obliquely to their carriers. Experiment 3 differed from Experiment 1 in that the subjects were asked to detect the presence of a subjective contour, rather than to discriminate left- and right-oblique subjective contours. This change was necessary to avoid confounding identification of the carrier orientation with identification of the orientation of the subjective contour. On half of the trials, a subjective contour was present, and on the other half, it was not.

There are interesting complexities in this experiment that concern the oblique effect (see, e.g., Vogels \& Orban, 1987). Consider panels $C$ and $E$ of Figure 5, which differ in contour/carrier orthogonality. If the orthogonal subjective contour panel in $C$ were found to be more salient than the nonorthogonal contour in panel $\mathrm{E}$, this would be consistent with an orthogonality bias. However, it could also reflect an oblique effect for subjective contours. If, to eliminate this interpretation, the pattern in panel $\mathrm{E}$ were rotated by $45^{\circ}$ and found to be less salient than the one in panel $\mathrm{C}$, this could represent an oblique effect at the level of the carrier, rather than reduced salience owing to carrier/contour nonorthogonality. A further complication is seen when comparing panels $C$ and $E$ of Figure 5. Let $a$ represent the distance between terminators when the subjective contour is orthogonal to the carrier grating (panel C). When the subjective contour is rotated $\alpha$ degrees from its orthogonal orientation, the separation between terminators becomes $a / \cos (\alpha)$; that is, as the orientation of the subjective contour approaches the orientation of the carrier, the separation between terminators increases. The change in terminator density with subjective contour orientation can be offset by a decrease in the carrier spatial frequency. Therefore, to assess the importance of contour/carrier orthogonality, three factors were manipulated in the construction of the stimuli in the present experiment. These were (1) carrier orientation (horizontal vs. oblique), (2) subjective contour orientation (vertical vs. oblique), and (3) carrier spatial frequency ( 3.0 and $2.14 \mathrm{cpd}$ ). Graphical depictions of a subset of these stimuli are given in Figure 5.

If there is a bias toward carrier/contour orthogonality, detection of a vertical subjective contour in a horizontal carrier should be easier than detection of an oblique subjective contour in a horizontal carrier. Furthermore, the detection of a left-oblique subjective contour in a rightoblique carrier should be easier than the detection of a vertical subjective contour on a right-oblique carrier. If the carrier/contour relationship is irrelevant to the de- 
A

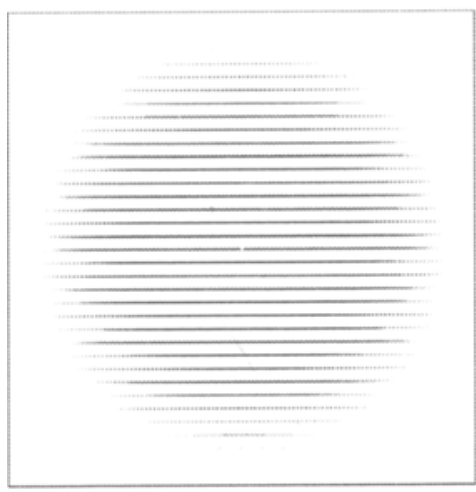

B

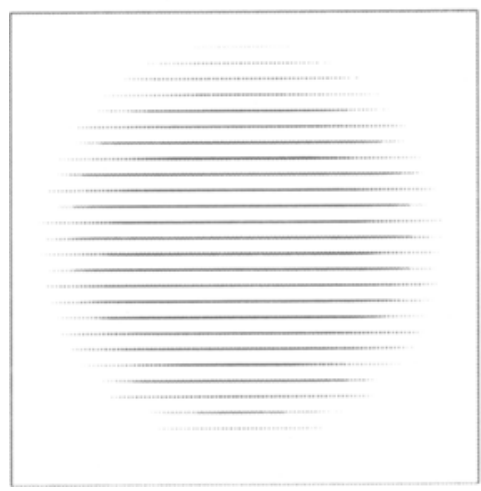

C

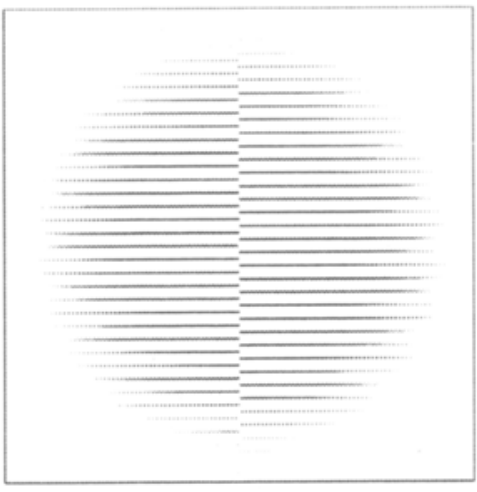

D

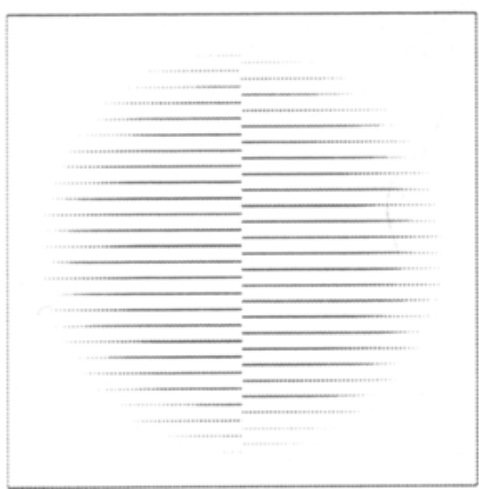

E

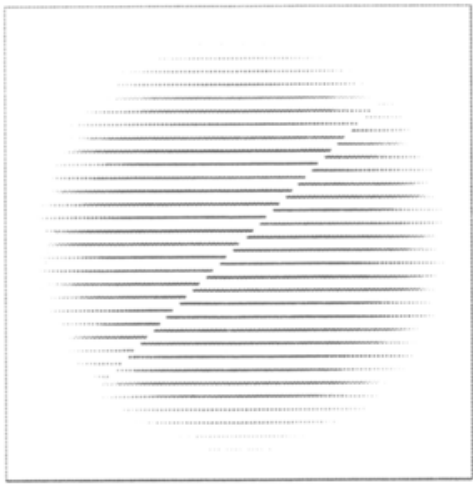

F

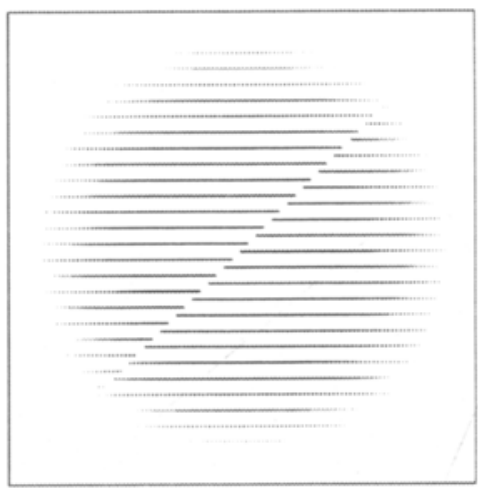

Figure 5. The stimuli used in Experiment 3. Panels $A$ and $B$ show the 3.0- and 2.14-cpd carrier gratings, respectively. Panels $C$ and $D$ show the orthogonal subjective contours, and panels $E$ and $F$ show the oblique subjective contours. To test conditions involving oblique carrier gratings, the computer monitor was rotated by $45^{\circ}$.

tection of a subjective contour, there would be no reason to expect this result to occur.

\section{Method}

Subjects. There were 11 subjects in Experiment 3. Only 1 had any previous experience in an experiment of this sort. All reported normal or corrected-to-normal vision.

Apparatus and Materials. As with Experiment 1, the stimuli were presented on an Ikagami monitor under the control of the Quadra 950 computer. The six stimuli depicted in Figure 5 were the only stimuli actually created by the computer. These involved horizontal carriers of two spatial frequencies and both vertical and oblique subjective contours. To create the second set of stimuli, the computer monitor was rotated by $45^{\circ}$. Rotating the monitor obviates difficulties associated with drawing diagonal lines on a raster grid.

Procedure. The experiment may be considered a 2 (carrier spatial frequencies) $\times 2$ (carrier orientations) $\times 2$ (subjective contour orientations) within-subjects design. Each of the eight experimental conditions was presented to each of the 11 subjects in a different random order. For each condition, there were seven SOAs (30, $45,60,75,90,105$, and $120 \mathrm{msec}$ ). A block of trials within a condition involved the presentation of the target-present and targetabsent stimuli at each of the seven SOAs. There were 15 replications of each block for each of the eight conditions. All the responses from one condition were collected before the next condition began. If necessary, the monitor was rotated between conditions. Each sub- ject was presented with 1,680 trials: 7 (exposure durations) $\times 8$ (conditions) $\times 2$ (responses, present/absent) $\times 15$ (blocks) $=1,680$.

\section{Results and Discussion}

The threshold data were submitted to a $2 \times 2 \times 2$ within-factor ANOVA. There was a significant effect of carrier orientation $[F(1,10)=43.69, p<.0001]$, indicating that horizontal carriers produced lower thresholds than did oblique carriers - that is, there was an oblique effect. There was a significant effect of carrier spatial frequency $[F(1,10)=18.14, p<.005]$, which, consistent with the results of Experiment 1, indicated that higher carrier frequencies produced higher detection thresholds than did lower carrier spatial frequencies. There was also a significant interaction between carrier orientation and subjective contour orientation $[F(1,10)=$ $21.02, p<.005]$. No other main effects or interactions were significant.

The interaction shown in Figure 6 favors the interpretation that the subjective contours oriented perpendicular to their carrier gratings are more easily detected than those oriented oblique to the carrier. Specifically, when the carrier was horizontal, thresholds were lower for ver- 

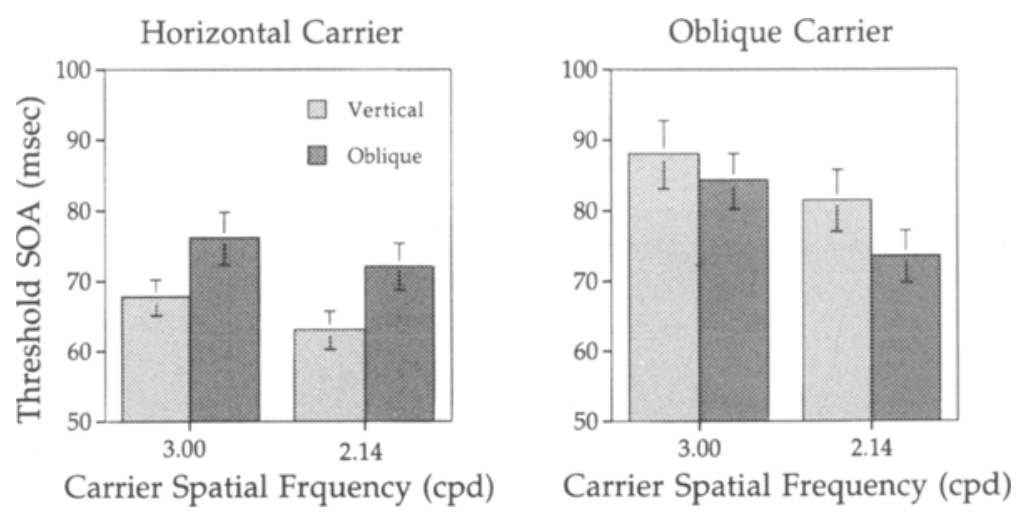

\begin{abstract}
Figure 6. The results of Experiment 3. Threshold stimulus onset asynchrony (SOA) is plotted as a function of carrier orientation, carrier spatial frequency, and subjective contour orientation. When the carrier is horizontal, the vertical contour is orthogonal to it; when the carrier is oblique $\left(+45^{\circ}\right)$, the oblique contour $\left(-45^{\circ}\right)$ is orthogonal to it. The critical result is that thresholds are lower when the carrier and the contour are orthogonal. That is, for the horizontal carrier, thresholds are lower for the vertical contours, and for the oblique carriers, thresholds are lower for the oblique contours. (Error bars indicate $\pm 1 S E M$.)
\end{abstract}

tical subjective contours than for oblique subjective contours. When the carrier was oblique, thresholds were lower for oblique subjective contours than for vertical subjective contours. This is in agreement with the model of von der Heydt and Peterhans (1989) and Soriano et al. (1996). We show below that the orthogonality effect may be attributable to the change in terminator density with changes in contour/carrier orientation.

\section{A MODEL}

This section develops a model of the results described in Experiments 1 and 3. The model makes the following two assumptions. First, subjective contours are encoded in a two-stage process, such as the one described in Figure 1. The first stage extracts line endings. This can be accomplished with end-stopped filters having a range of orientation and spatial frequency selectivities. The strength of an end-stopped response depends on the interline spacing (spatial frequency) in the carrier grating.

The second assumption is that second-layer filters covering a range of orientations are applied to the endstopped filters. The spatial extent of a second-layer filter is tied to the spatial frequency tuning of the end-stopped filters from which it draws its input (see, also, Wilson \& Richards, 1992).

Figure 7 provides a depiction of how the size of the second-layer filter is related to the frequency of the carrier grating. High-frequency carriers induce optimal activity in end-stopped cells having small spatial extent. Therefore, the second-layer filter connected to these endstopped cells also has relatively small spatial extent (panels $A$ and $B$ ). The response of the second-layer filter in panels $A$ and $B$ would be independent of aperture size. Low-frequency carriers induce optimal activity in endstopped cells having larger spatial extent, and thus, the second-layer filter connected to these has larger spatial extent (panels $C$ and $D$ ). The response of the second-layer filter in panels $C$ and $D$ depends on aperture size; more terminators fall within its receptive field in panel $D$ than in panel $\mathrm{C}$.

To model the results of the experiments described above, it is assumed that the sensitivity of second-layer filters (whose responses determine detection and discrimination thresholds) is inversely proportional to threshold SOA; short-SOA thresholds indicate high sensitivity, and long-SOA thresholds indicate low sensitivity. ${ }^{2} \mathrm{We}$ assume the existence of many second-layer filters (having the characteristics just described), and the most sensitive second-layer filter determines threshold. (The possible role of probability summation is not considered here.) Therefore, the data to be fit are the inverses of the threshold SOAs; sensitivity $=c / T_{\text {SOA }}$, where $c$ is a scaling constant. We assume that sensitivity is related to the strength of the second layer mechanism's response (SLR) to the stimuli.

In Experiment 1, the subjects had to decide, on each trial, whether the subjective contour presented was a left or a right oblique. The left panel of Figure 8 replots the data from Figure 6 in terms of sensitivity. For the two largest aperture sizes, sensitivity declines smoothly as carrier frequency increases. The lack of influence of aperture size in these two cases suggests that the smooth loss of sensitivity reflects reduced salience of the carrier. Thus, the responses of an end-stopped mechanism to line endings may be represented as a low-pass function of spatial frequency $(\omega)$, as expressed in Equation 1:

$$
T(\omega)=e^{-\omega / \sigma^{2}} .
$$

Equation 1 indicates that terminators in a low-frequency carrier provide a stronger signal (for a given carrier contrast) than do terminators in a high-frequency carrier. 
A

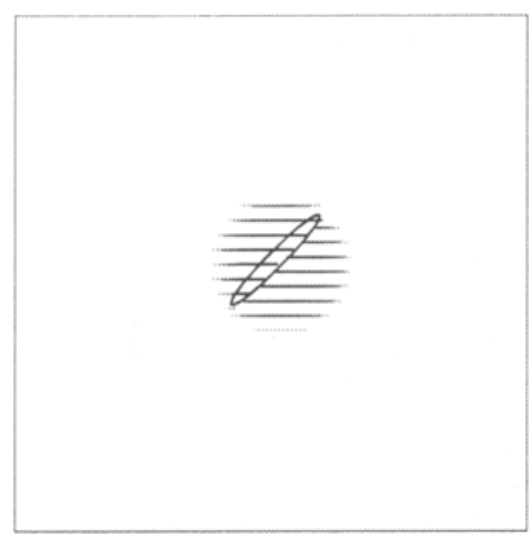

B

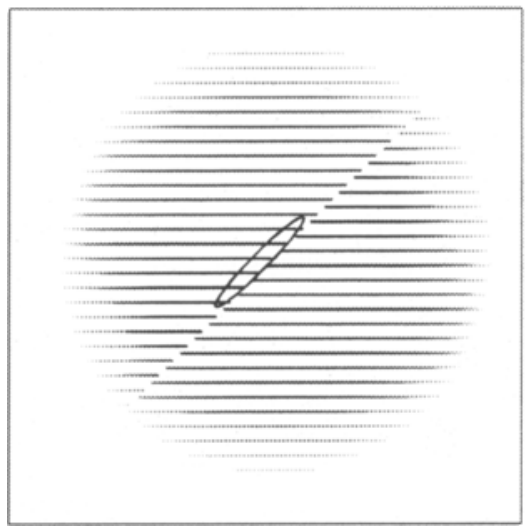

C

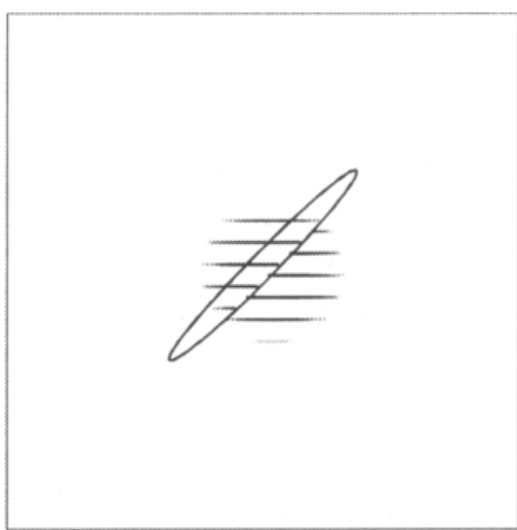

D

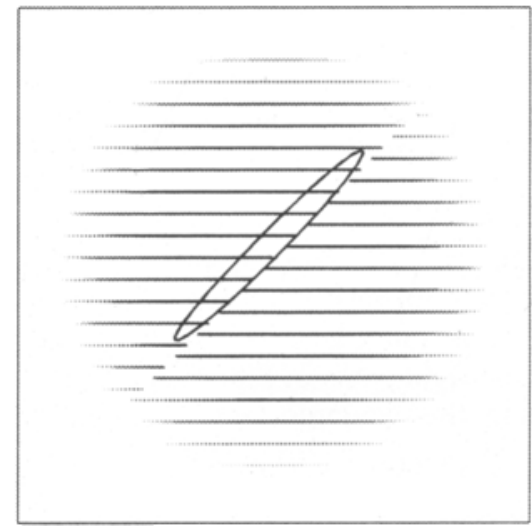

Figure 7. The relationship between second-layer filter size and carrier spatial frequency. Many models assume that there is a relationship between the size of the second-layer filter and that of the first-layer filters on whose outputs it operates. The consequence of this relationship is shown for two aperture sizes and two carrier spatial frequencies. Panels $A$ and $B$ show small and large apertures containing relatively high-frequency carriers. The aperture in panel $A$ is large enough to maximally stimulate the second-layer filter operating on it. The aperture in panel $B$ is larger, but there is equivalent stimulation of the second-layer filter. Panels $C$ and $D$ show small and large apertures containing relatively low-frequency carriers. The aperture in panel $\mathbf{C}$ is not large enough to maximally stimulate the second-layer filter operating on it, whereas the aperture in panel $D$ is. Therefore, the conditions depicted in panels $A$ and $B$ would be expected to lead to similar thresholds, whereas higher thresholds would be expected for the condition depicted in panel $C$ than for that depicted in panel $D$.

The model assumes a second-layer filter having a size proportional to the scale of the end-stopped mechanisms from which it draws its input. For a particular carrier frequency, the response of the second-layer filter increases as the length of a subjective contour increases (i.e., as the aperture size increases). Once the length of the subjective contour exceeds the spatial extent of the secondlayer receptive field, however, its response can increase no further. The SLR is represented by Equation 2:

$$
\operatorname{SLR}=\min [\beta T(\omega), \gamma T(\omega)] .
$$

$\beta$ represents the number of cycles of the carrier in the stimulus. $\gamma$ acts as a saturating threshold that specifies the receptive field size of the second-layer filter (in terms of the number of cycles of the carrier). According to
Equation 2, SLR increases as $\beta$ increases, then asymptotes at the point where $\beta=\gamma$.

Given the assumptions that (1) the size of a secondlayer filter is linked to the size of the end-stopped filters from which it draws its input and (2) the scale of the optimally stimulated end-stopped filters changes with carrier spatial frequency, it is only necessary to derive values for $\sigma$ and $\gamma$ to match the sensitivity data shown in the left panel of Figure 8. Using a least-square minimization procedure, values of $\sigma=1.95$ and $\gamma=5.35$ were found to provide an almost perfect fit to the data shown in the left panel of Figure $8\left(r^{2}=.97\right)$. The value of $\gamma=5.35$ is the number of cycles of the carrier falling within the receptive field of the second-layer filter. Therefore, $\gamma / \omega$ represents the spatial extent of the second-layer filter; if 

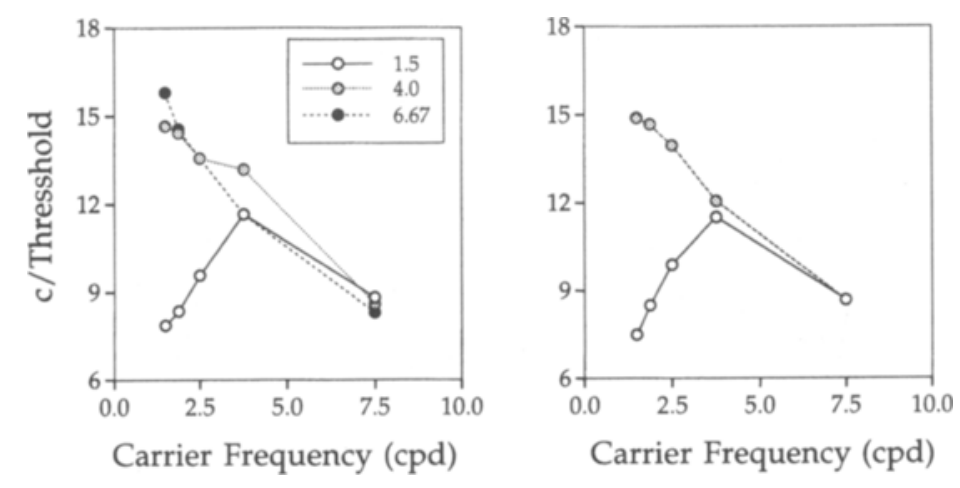

Figure 8. A comparison of the obtained and simulated second-layer responses for Experiment 1. The left panel replots the data of Figure 3 as $c /$ threshold for the three aperture sizes and the five carrier frequencies. The right panel shows the responses of the model. The model captures the most prominent features of the data. For the two largest apertures, sensitivity is independent of aperture size and shows a low-pass dependence on carrier spatial frequency. For the smallest aperture, sensitivity increases with carrier spatial frequency (because smaller second-layer filters are engaged by higher carrier frequencies) but then declines again from 3.75 to $7.5 \mathrm{cpd}$.

$\omega=2 \mathrm{cpd}$, the spatial extent of the second-layer filter is $5.35 / 2 \approx 2.68^{\circ}$. The model responses were scaled (to bring them into the same range as the measured sentivities) and plotted in the right panel of Figure 8. Note that, in the sensitivity data, the two largest apertures yielded virtually identical results and that, in the model, they are indistinguishable. Thus, two simple assumptions yield a model that provides an excellent fit to the data.

The results of Experiment 3 demonstrated an orthogonality advantage for contour/carrier relationships. There are a number of reasons why such an advantage might occur. An obvious possibility is that the orthogonality advantage reflects an orthogonality relationship in the orientation sensitivities of the first- and second-layer filters; that is, first- and second-layer filters are tuned to orthogonal orientations, as in Figure 1. The von der Heydt and Peterhans (1989) model makes an assumption of this sort. A less categorical possibility is that second-layer filters receive the strongest input from orthogonally oriented first-layer filters and that the strength of the input decreases as the orientations of the first- and the secondlayer filters become more similar. It is interesting to note that this relationship is a direct consequence of the fact that, for a given carrier spatial frequency, the separation between terminators-determined by the wavelength $\left(\omega^{-1}\right)$ of the carrier-is smallest when the contour and the carrier are orthogonal and increases as

$$
\omega^{-1 / \cos (\alpha)}
$$

where $\alpha$ is the angular deviation from orthogonality. Thus, for an aperture of fixed size, the number of terminators within the aperture is maximal when the contour/carrier relationship is orthogonal and decreases monotonically with deviations from orthogonality. In Experiment 1 , the subjective contour was oriented $45^{\circ}$ to the carrier. Had the subjective contour been orthogonal to the carrier, there would have been $41 \%$ more terminators within the second-layer receptive field than in the oblique condition.

The results of Experiment 3 showed an overall main effect of carrier orientation; the horizontal carriers produced shorter SOA thresholds than did the oblique carriers. Therefore, to apply the preceding analysis to the results of Experiment 2, the effect of carrier orientation must be taken into account. The carrier effect may be characterized as a limitation imposed by the first-layer filters, reflecting well-documented oblique effects (Appelle, 1972). Assuming that horizontal and vertical gratings are encoded with equal efficiency, the relative sensitivities to gratings of different orientations could be expressed as a cosine function of orientation that goes through one cycle in $90^{\circ}$; horizontal and vertical would be at the peaks of the cosine, and $\pm 45^{\circ}$ would be at the troughs:

$$
T(\omega, \theta)=\mu[1+c \cos (4 \theta)] T(\omega) .
$$

The parameters $\mu$ and $c$ define the mean and contrast, respectively, of the cosine modulation and determine the relative responses of the first-layer filters to gratings of different orientations. Equation 4 shows that the sensitivity of the first-layer filters and, hence, the responses to line endings are limited by both the spatial frequency $(\omega)$ and the orientation $(\theta)$ of the carrier.

As before, the spatial frequency of the carrier determines the size of the second-layer filter that governs performance. All the stimuli used in Experiment 2 were larger than the size of the second-layer filter $\left(\gamma \omega^{-1}\right.$, where 

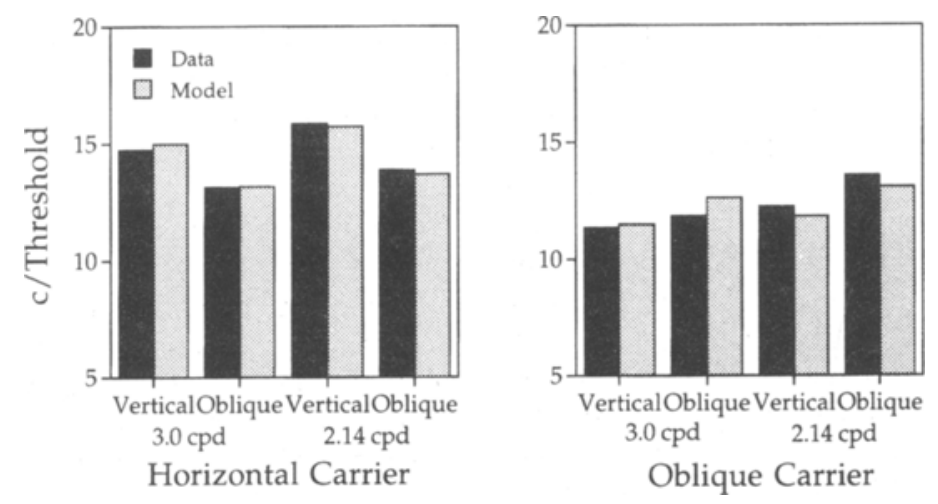

Figure 9. A comparison of the obtained and simulated second-layer responses for Experiment 3. Black bars replot the data of Figure 6 as $c /$ threshold. The gray bars plot the model responses. Overall, the model provides an excellent account of the data. The only discrepancy is that the model predicts higher sensitivity to the orthogonal condition for the highfrequency oblique carrier than is observed in the actual data.

$\omega^{-1}$ is the wavelength of the carrier grating) deduced from the results of Experiment 1. Equation 3 shows that, for a fixed carrier wavelength $\left(\omega^{-1}\right)$, the separation between terminators increases as $\omega 1 / \cos (\alpha)$, where $\alpha$ is the angular deviation from orthogonality. Therefore, the density of terminators in some unit area (i.e., the receptive field) decreases as $\cos (\alpha)$. Equation 5 expresses the strength of the second-layer response as a function of carrier frequency $(\omega)$ and orientation $(\theta)$ and the angular deviation $(\alpha)$ of contour/carrier relationship from orthogonality ( $\alpha=0$, when the carrier and contour are orthogonal):

$$
\operatorname{SLR}=\gamma \cos (\alpha) T(\omega, \theta) .
$$

The only parameters to be derived are $\mu$ and $c$ from Equation 4, which control the relative salience of terminators in the horizontal and oblique carriers. With $\gamma$ and $\sigma$ fixed $^{3}$ (from the analysis for Experiment 1), a leastsquares minimization procedure was used to find values of $\mu$ and $c$ that provide the best fit of Equation 5 to the data of Experiment 2. The best-fitting values of $\mu$ and $c$ were .38 and .23 , respectively. The model results were scaled and plotted in Figure 9, along with the actual sensitivity data of Experiment 3 . The model provides an excellent fit to the data $\left(r^{2}=.93\right)$. The largest discrepancy is seen in the case of the 3.0-cpd oblique carrier with an orthogonal subjective contour (oblique). The psychophysical sensitivity in this condition is slightly lower than predicted by the model. Despite this difference, the model is in excellent agreement with the qualitative features of the data. (Note that if we allow $\sigma$ to differ from the model of Experiment 1, an $r^{2}=.97$ can be obtained.)

\section{GENERAL DISCUSSION}

Experiments 1 and 3 were conducted to refine our understanding of the mechanisms that encode subjective contours. Experiment 1 showed that subjective contour discrimination depends on both carrier spatial frequency and subjective contour length (aperture size). This result is well explained by a two-stage process in which a second-layer filter integrates the responses of end-stopped mechanisms to the terminators defining the subjective contour. In the model, (1) the end-stopped mechanisms have low-pass sensitivity to carrier spatial frequency, and (2) the size of the second-layer filter is proportional to the scale of the end-stopped mechanisms that respond optimally to the terminators. Experiment 3 showed that the detectability of subjective contours is determined by carrier spatial frequency, carrier orientation, and the orientation difference between the contour and the carrier. The results of Experiment 3 are interpretable in terms of the same principles used to interpret the results of Experiment 1 . The model does not assume a privileged connection between orthogonally oriented first- and secondlayer filters to explain the contour/carrier orthogonality effect. According to the model, the contour/carrier orthogonality effect reflects the fact that orthogonally oriented terminators are more densely packed within the second-layer receptive field than are terminators of other orientations.

In many respects, the results of Experiments 1,2, and 3 are in agreement with past research, especially that of von der Heydt and Peterhans (1989) and Soriano et al. (1996).

\section{Asymptotic Contour Length}

A main empirical result of Experiment 1 was that the discriminability of subjective contours was unaffected by aperture size when the apertures were sufficiently large. This is consistent with the physiological results of von der Heydt and Peterhans (1989, Figures 12 and 13). Their data show that contour cells increased their firing as the number of line endings in the subjective contour increased, up to a point, after which the addition of further lines did not affect the cell's firing rate. On average, a cell's response asymptotes when there are 12 line endings within its receptive field. This finding is in reasonable agreement with the conclusions drawn from the pres- 
ent model. When the contour and the carrier are orthogonal, our model indicates that there should be about 7.57 cycles of the carrier within the second-layer receptive field. A phase shift of $180^{\circ}$ would produce $2 * 7.57=$ 15.14 terminators within the second-layer receptive field.

In their psychophysical study, Soriano et al. (1996) found that rated salience of subjective contours asymptoted when the contour was defined by 10 terminators. This number is in good agreement with the present study and with the physiological results of von der Heydt and Peterhans (1989). There is an interesting difference between the model described by von der Heydt and Peterhans and the one described in Figure 1. The model in Figure 1 integrates end-stopped responses on only one side of the subjective contour, whereas the von der Heydt and Peterhans model integrates end-stopped responses on both sides of the subjective contour. Their physiological data do not allow us to determine which of these two alternatives is more plausible.

\section{Contour/Carrier Orthogonality}

Experiment 3 demonstrated that detection of subjective contours is best when the contour and the carrier are orthogonal. This is in general agreement with von der Heydt and Peterhans's (1989) report that six of eight contour cells tested responded best when the contour and the carrier were orthogonal. As was mentioned earlier, Soriano et al. (1996, Figure 8) examined displays in which the carrier on one side of the contour was orthogonal to it, whereas the carrier on the opposite side met the contour at an oblique angle. They found that the rated salience of subjective contours decreased as the orientation difference between the gratings on either side of the contour increased, indicating an orthogonality advantage. However, the present model suggests a different interpretation. In their displays, Soriano et al. kept the number of terminators constant on both sides of the subjective contour; according to the present model, it is change in terminator density that accounts for the contour/carrier orthogonality advantage. However, varying the orientation of one of the gratings required a concomitant change in its spatial frequency. Our results suggest that both high carrier frequencies and oblique carriers produce less salient subjective contours. Therefore, the orthogonality effect observed by Soriano et al. may actually have been a carrier spatial frequency or orientation effect.

\section{Carrier Orientation}

Soriano et al. (1996, Figure 10) found that subjective contours were most salient when the carriers were horizontal, slightly less salient when they were vertical, and weakest when they were oblique $\left( \pm 45^{\circ}\right)$. This is consistent with the results of Experiment 3, which showed that the detectability of subjective contours was generally impaired when carriers were oblique. The fact that horizontal and vertical carriers did not produce equally salient subjective contours is slightly inconsistent with the assumption (expressed in Equation 4) that horizontal and vertical carriers should be equally detectable. If the advantage for horizontal over vertical carriers were to be found in the present task, the model would need only a minor modification of Equation 4.

\section{Carrier Frequency}

The main inconsistency between the present data and those reported by Soriano et al. (1996) concerns the effect of carrier frequency on the detectability of subjective contours. Soriano et al. (Figure 6) showed that the rated salience of subjective contours declined more or less monotonically with carrier frequency from about 10 to $0.33 \mathrm{cpd}$. In other words, the rated salience of subjective contours showed a high-pass dependence on spatial frequency. By contrast, our subjects found subjective contours defined in high-frequency carriers harder to detect and to discriminate (Experiments 1 and 3 ) than those defined in low-frequency carriers. Furthermore, most of the subjects in Experiment 2 judged subjective contours to be stronger as the carrier frequency decreased from 7.5 to $1.5 \mathrm{cpd}$. Thus, in three separate tasks (detection, discrimination, and ratings) with different groups of subjects, we found evidence that subjective contours are more salient as carrier frequency decreases. The consistency of our findings concerning carrier frequency and the discrepancy with Soriano et al. is puzzling, and we have no ready explanation for this difference. Certainly, the presentation conditions and stimulus structures were different, and this may have played a role in the different patterns of results. We also note that Soriano et al. employed a rating task, and subjects may have responded to demand characteristics in the stimulus set. This is one possible explanation for the two patterns of responding that we observed in Experiment 2.

Although our results are inconsistent with those of Soriano et al. (1996) and von der Heydt and Peterhans (1989), they are partially consistent with those of Lesher and Mingolla (1993). Lesher and Mingolla created Kanizsa-type subjective contours of various sizes (Shipley \& Kellman, 1992 ), defined by concentric, $270^{\circ}$ arcs. Both the number and the widths of the arcs were varied. Subjects rated the clarity and brightness of subjective figures formed in this manner. A principal finding was that both rated clarity and brightness followed an inverted-U-shaped function of line density. At large and small arc spacings, rated clarity and brightness were lower than at intermediate spacings. Lesher and Mingolla suggested that the reduced salience of subjective contours at small arc spacings reflected interference in the encoding of line endings. They pointed out that an end-stopped mechanism with inhibitory side-lobes (see Figure 1 and Dobbins, Zucker, \& Cynader, 1989) would decrease its response when additional carrier lines encroached upon these regions. That is, if the end-stopped mechanisms are spatial frequency selective, they should show low-pass or bandpass sensitivity to the frequency of the carrier gratings. This is essentially the explanation we gave for the lowpass sensitivity to carrier frequency found in our Exper- 
iments 1, 2, and 3. Our data are inconsistent with those of Lesher and Mingolla, in that they found reduced salience at small arc spacings only when arc width was greater than 1 pixel. When arc width was 1 pixel, the salience of the subjective contour increased monotonically with line density. Thus, for some reason, Lesher and Mingolla found high-pass sensitivity to the "carriers" (concentric arcs) for arc widths of 1 pixel (consistent with Soriano et al., 1996), whereas the results of the three experiments reported here generally indicate low-pass sensitivity to carrier frequencies. These discrepancies may have to do with differences in the tasks employed (ratings vs. detection and discrimination) and/or the contrasts in the stimuli ( $>90 \%$ vs. $25 \%$ in the present experiments).

Our model provides a very succinct account of the data and is in general agreement with many aspects of previously reported data, but alternative explanations are possible. Earlier, we considered the possibility that Experiment 1 might be interpreted in terms of terminator detection. If the subjects simply detected individual terminators and their positions in the aperture, the orientation of the subjective contour could have been inferred.

Such an account does not explain the U-shaped threshold function for the smallest aperture. For the two largest apertures, the data indicated an inverse relationship between carrier spatial frequency and sensitivity. If the results of Experiment 1 involved only the localization of terminators, performance should be monotonically related to carrier frequency in all conditions; no U-shaped function should have been observed. Furthermore, a terminator detection explanation would predict that sensitivity should increase with aperture size at all carrier frequencies, because terminators would appear further from the center of the display and, therefore, would more accurately convey subjective contour orientation. This prediction fails, in general. We conclude that the terminator localization account of Experiment 1 would need further assumptions before it could explain all aspects of the data set, whereas all such data are nicely explained by the two-stage model.

An interpretation of Experiment 3 might also be attempted in terms of terminator detection. Rather than appealing to the changes in terminator density with changes in the relative orientations of carrier and contour, one could argue that line endings that meet the subjective contour at an oblique angle are signaled more weakly than line endings orthogonal to the subjective contour. On this account, there may be no need to integrate the responses of terminators by a second-layer filter, as we have suggested. This interpretation of Experiment 3 cannot be discounted, but it is not clear how to examine it empirically. The data require an explanation of the effects of carrier orientation and spatial frequency, as well as of the relative orientations of the carrier and the contour. In defense of the model presented here, we note that a few simple assumptions go a long way toward explaining an extensive set of data (Experiments 1, 2, and 3). It is not obvious that an alternative - and equally simple - set of assumptions based on terminator detection and localization could explain the entire set of data.

Experiment 3 showed a contour/carrier orthogonality advantage. Although this result is consistent with past work (reviewed earlier), it is apparently inconsistent with the work of Gillam (1987). Gillam reported that subjective contours are stronger when randomly oriented lines co-terminate than when lines orthogonal to the subjective contour co-terminate. It was also reported that subjective contours were stronger when the carrier and the contour were nonorthogonal. The stimuli in these tasks were lines cut by white space, rather than phase-shifted gratings such as those used in the present task (see, also, Lesher \& Mingolla, 1993). Although Gurnsey and von Grünau (1997) showed that these two situations can give rise to similar perceptual judgments about motion, the percepts in the static case may diverge. To further investigate the relationship between the present work and Gillam's, it would be important to put Gillam's stimuli into the same kind of discrimination or detection task as those used in Experiments 1 and 3. At this point, other than noting task and stimulus difference, we have no com pelling explanation for the differences between her results and ours.

We conclude that the kind of two-stage model proposed by Grossberg and Mingolla (1985), Gurnsey and von Grünau (1997), von der Heydt and Peterhans (1989), and Wilson and Richards (1992) provides a very elegant explanation of the detection and discrimination of subjective contours defined by offset gratings. Such models are consistent with existing data showing that subjective contours are easily discriminated (Gurnsey et al., 1992) by humans and cats (Bravo, Blake, \& Morrison, 1988). These models operate entirely in the spatial domain and do not require motion energy in the stimulus to encode the orientations of subjective contours (cf. Skottun, 1994).

\section{REFERENCES}

APPELle, S. (1972). Perception and discrimination as a function of stimulus orientation: the oblique effect in man and animals. $P$ sychological Bulletin, 78, 266-278.

BERGEN, J. R., \& LANDY, M. S. (1991). Computational modeling of visual texture segregation. In M S. Landy \& J. A. Movshon (Eds.), Computational models of early visual processing (pp. 253-271). Cambridge, MA: MIT Press.

Bradley, D. R., \& Dumais, S. T. (1975). Ambiguous cognitive contours. Nature, 257, 582-584

Bravo, M., Blake, R., \& Morrison, S. (1988). Cats see subjective contours. Vision Research, 28, 861-865.

Coren, S. (1972). Subjective contours and apparent depth. Psychological Review, 79, 359-367.

Davis, G., \& Driver, J. (1994). Parallel detection of Kanizsa subjective figures in the human visual system. Nature, 371, 791-793.

Dobbins, A., ZuCker, S. W., \& CYnader, M. S. (1989). Endstopping and curvature. Vision Research, 29, 1371-1387.

Gillam, B. (1987). Perceptual grouping and subjective contours. In S. Petry \& G. E. Meyer (Eds.), The perception of illusory contours (pp. 268-273). New York: Springer-Verlag.

GrEgory, R. L. (1972). Cognitive contours. Nature, 238, 51-52. 
Grossberg, S., \& Mingolla, E. (1985). Neural dynamics of perceptual grouping: Textures, boundaries, and emergent segmentations. Perception \& Psychophysics, 38, 141-171.

GurnSEy, R., Herbert, A., \& Kenemy, J. (1998). Bilateral symmetry embedded in noise is detected accurately only at fixation. Vision Research, 38, 3795-3803.

Gurnsey, R., Humphrey, G. K., \& Kapitan, P. (1992). Parallel discrimination of subjective contours defined by offset gratings. Perception \& Psychophysics, 52, 263-276.

Gurnsey, R., Pearson, P., \& DAY, D. (1996). Texture discrimination along the horizontal meridian: Effects of magnification, frequency content and micropattern orientation. Journal of Experimental Psychology: Human Perception \& Performance, 22, 738-757.

GURnSEY, R., POIRIER, F., \& GASCON, E. (1996). There is no evidence that Kanizsa-type subjective contours can be detected in parallel. Perception, 25, 861-874.

GURNSEY, R., \& VON GRÜNAU, M. (1997). Illusory contour-motion arising from translating terminators. Vision Research, 37, 1007-1024.

Heitger, F., Rosenthaler, L., von der Heydt, R., Peterhans, E., \& KUBLER, O. (1992). Simulation of neural contour mechanisms: From simple to end-stopped cells. Vision Research, 32, 963-978.

KANIZSA, G. (1987). Quasi-perceptual margins in homogeneously stimulated fields. In S. Petry \& G. E. Meyer (Eds.), The perception of illusory contours (pp. 43-49). New York: Springer-Verlag.

KATO, H., Bishop, P. O., \& OrBAN, G. (1978). Hypercomplex and simple/complex cell classifications in cat striate cortex. Journal of Neurophysiology, 41, 1071-1095.

KEPPEL, G. (1991). Design and analysis: A researcher's handbook. Englewood Cliffs, NJ: Prentice-Hall.

LeShER, G. W., \& MingOlla, E. (1993). The role of edges and line-ends in illusory contour formation. Vision Research, 33, 2253-2270.

MARR, D. (1982). Vision. San Francisco: Freeman.

Rock, I., \& ANSON, R. (1979). Illusory contours as the solution to a problem. Perception, 8, 665-681.

Shipley, T. F., \& KellmaN, P. J. (1992). Strength of visual interpolation depends on the ratio of physically specified to total edge length. Perception \& Psychophysics, 52, 97-106.

Skottun, B. C. (1994). Illusory contours and linear filters. Experimental Brain Research, 100, 360-364.

Soriano, M., Spillman, L., \& BaCH, M. (1996). The abutting grating illusion. Vision Research, 36, 109-116.
VoGels, R., \& Orban, G. (1987). Illusory contour orientation discrimination. Vision Research, 27, 453-467.

yon DeR Heydt, R., \& Peterhans, E. (1989). Mechanisms of contour perception in monkey visual cortex: I. Lines of pattern discontinuity. Journal of Neuroscience, 9, 1731-1748.

WILSON, H. R., \& MAST, R. (1993). Illusory motion of texture boundaries. Vision Research, 33, 1437-1446.

Wilson, H. R., \& RichaRds, W. A. (1992). Curvature and separation discrimination at texture boundaries. Journal of the Optical Society of America A, 9, 1653-1662.

\section{NOTES}

1. According to the top-down view, subjective contours result from perceptual organization of the display, which is a central, conceptually driven process guided by memory, perceptual set, expectations, cognitive strategies, and selective attention. Following Kanizsa (1987), major theories of this type have been proposed by Coren (1972), Gregory (1972), Rock and Anson (1979), and Bradley and Dumais (1975). Topdown theories of subjective contours have typically employed stimuli originally studied by Kanizsa, rather than the offset grating stimuli used here. Davis and Driver (1994) attempted to show that Kanizsa-type subjective contours are encoded in a bottom-up manner, but this result remains ambiguous (Gurnsey, Poirier, \& Gascon, 1996).

2. The SOA at which an orientation discrimination or detection can be made does not necessarily reflect the strength of a subjective contour; it could simply reflect the time taken to construct the necessary representation. However, in our view, the very good agreement between the rating data of Experiment 2 (Figure 4A) and the threshold data of Experiment 1 (Figure 3 ) justıfies the assumption that threshold SOA is a good measure of subjective contour strength, and not simply a reflection of contour dynamics.

3. Note that $\gamma$ was set to $5.35 / \cos (\pi / 4)=7.57$. In the analysis of Experiment $1, \gamma$ was determined to be 5.35 cycles of the carrier, when the carrier was oriented $45^{\circ}$ to the contour. If the carrier had been oriented perpendicular to the contour, there would have been about $\cos (\pi / 4)^{-1}$, or $41 \%$, more cycles of the carrier within the second-layer receptive field.

(Manuscript received July 15, 1997; revision accepted for publication August 5, 1998.) 